\title{
Platinum-based Doublet Chemotherapy for Advanced Gastric Cancer with Disseminated Intravascular Coagulation
}

\author{
KEIJI SUGIYAMA ${ }^{1}$, YUKIYA NARITA ${ }^{1}$, SHIGENORI KADOWAKI ${ }^{1}$, \\ TAKASHI URA ${ }^{1}$, MASAHIRO TAJIKA $^{2}$ and KEI MURO ${ }^{1}$ \\ Departments of ${ }^{1}$ Clinical Oncology and ${ }^{2}$ Endoscopy, Aichi Cancer Center Hospital, Aichi, Japan
}

\begin{abstract}
Background: Disseminated intravascular coagulation (DIC) is a rare complication of advanced gastric cancer (AGC). Despite reports of the efficacy of chemotherapy for AGC with DIC, little is known of platinum-based doublet therapy. Patients and Methods: We conducted a single-institute, retrospective chart review of 500 consecutive chemotherapynaïve patients with advanced gastric adenocarcinoma (recurrent or metastatic) from November 2010 to November 2015. Results: Six patients were diagnosed with AGC with DIC (1.2\%); five (1.0\%; 3 men, 2 women) received platinum-based doublet chemotherapy. All patients exhibited improved DIC and thrombocytopenia and survived for $>100$ days (range $=114-313$ ) with no therapy-related mortality. Grade $\geq 3$ adverse effects included neutropenia, anemia, hyponatremia, catheter-related infection and diarrhea (maximum: 2 patients each). Conclusion: Fluoropyrimidine plus platinum combination therapy was effective against DIC and yielded acceptable survival outcomes. Combination chemotherapy should be considered as a primary therapy for AGC with DIC.
\end{abstract}

Disseminated intravascular coagulopathy (DIC), characterized by clotting activation in small vessels and platelet and coagulation factor consumption leading to organ failure or fatal hemorrhage (1), can be caused by various diseases or severe conditions, including trauma, infectious disease, obstetric complications and malignancies. DIC is considered a rare complication of chemotherapy-naïve advanced gastric cancer (AGC) with an incidence rate of $1.6 \%$ (2). Although AGC with DIC is associated with an extremely poor outcome in the absence of cancer-specific

Correspondence to: Yukiya Narita, Department of Clinical Oncology, Aichi Cancer Center Hospital, 1-1 Kanokoden, Chikusaku, Nagoya, Aichi 464-8681, Japan. Tel: +81 527626111, Fax: +81 527528390, e-mail: yukiya.narita@aichi-cc.jp

Key Words: Chemotherapy, cisplatin, disseminated intravascular coagulopathy, fluoropyrimidine, 5-fluorouracil, gastric cancer, oxaliplatin, S- 1 . therapy, several recent reports demonstrated that 5fluorouracil $(5-\mathrm{FU}) /$ leucovorin $(3,4)$ or 5 -FU/methotrexate chemotherapy (2) were somewhat effective for AGC with DIC. However, despite the use of chemotherapy, the prognosis of AGC with DIC remained poor.

Although the standard chemotherapy regimen for AGC comprises fluoropyrimidine plus a platinum-based agent, little is known about the effect of platinum-based doublet chemotherapy for AGC with DIC. Accordingly, our present series of cases, involving fluoropyrimidine plus platinumtreated AGC with DIC, aims to assess the clinical benefits and safety profile of platinum-based doublet therapy for chemotherapy-naïve AGC with DIC and review the clinical courses of the identified patients.

\section{Patients and Methods}

We reviewed 500 consecutive chemotherapy-naïve patients with advanced gastric adenocarcinoma (recurrent, metastatic or unresectable) treated at Aichi Cancer Center Hospital, Japan, from November 2010 to November 2015. For this study, only subjects with DIC who received platinum-based doublet therapy were included. We analyzed the subjects' clinical features and courses, toxicity assessments, as well as clinical outcomes, including overall survival (OS) and time to treatment failure (TTF). Therapy-related toxicity assessments were performed according to the Common Terminology Criteria for Adverse Events, version 4.0.

DIC or coagulopathy was identified using the 1988 criteria of the Japanese Ministry of Health and Welfare (5); cases were scored according to underlying disease, bleeding symptoms, organ symptoms, elevated serum fibrin degradation product (FDP), decreased serum fibrinogen and prolonged prothrombin time. A score of $\geq 7$ points was considered definitive DIC, whereas at 5 or 6 points DIC or developing coagulopathy was suspected if other diseases were ruled out. DIC improvement was defined as a score decrease to $<5$.

\section{Results}

Patients' characteristics. Six patients were diagnosed with AGC with DIC (1.2\%) and five (1.0\%; 3 men, 2 women) received platinum-based doublet chemotherapy: $\mathrm{S}-1$ (tegafur/gimeracil/oteracil) plus cisplatin (SP or CDDP; $\mathrm{n}=2$ ) and oxaliplatin, leucovorin, as well as 5-FU (mFOLFOX6; 
$\mathrm{n}=3$ ). The remaining patient received non-platinum doublet chemotherapy (weekly paclitaxel therapy) and developed an early post-radical gastrectomy recurrence while receiving S1 adjuvant chemotherapy. The clinicopathological features of the five patients treated with platinum-based doublet chemotherapy are provided in Table I. The median age was 50 years (range $=35-66$ ) and three, one and one patients had Eastern Cooperative Oncology Group performance statuses of 1,2 and 4, respectively. All patients received diagnoses of undifferentiated adenocarcinoma and bone metastasis, while three and two had metastatic and recurrent disease, respectively. Immunohistochemically, three and two patients had a negative or undetermined human epidermal growth factor receptor 2 (HER2) status, respectively. The median platelet count was $2.7 \times 10^{4} / \mu$ l (range $\left.=0.5-11.3\right)$.

Outcomes of chemotherapy. All patients recovered from DIC after a median interval of 54 days $($ range $=11-92$ ) with a median time to an improved platelet count (more than $12 \times 10^{4} / \mu \mathrm{l}$ ) of 14 days (range $=7-54$ ). Reported grade $\geq 3$ adverse effects included neutropenia $(n=2)$, anemia $(n=1)$, hyponatremia $(n=2)$, catheter-related infection $(n=1)$ and diarrhea $(n=1)$. No therapy-related mortality was observed. Four of five patients (80\%) developed DIC after transient improvement with a median time to re-development of DIC of 83 days (range $=15-225$ ). The outcomes of chemotherapy are summarized in Table II.

\section{Case Reports}

Case 1. A 66-year-old male presented with low back pain and a history of distal gastrectomy for gastric adenocarcinoma 4 years earlier. Spinal computed tomography (CT) revealed multiple osteolytic lesions. Thrombocytopenia and elevated FDP emerged during radiation therapy for bone metastasis; he was diagnosed with gastric cancer-associated coagulopathy (pre-DIC). After completing radiation therapy, he received SP and achieved confirmed platelet count recovery and improved DIC at days 41 and 92, respectively. After 3 cycles of SP therapy, CT revealed a new bone lesion. He subsequently received weekly paclitaxel as second-line chemotherapy for confirmed progressive disease but developed a recurrence of DIC and died 191 days after the initiation of first-line chemotherapy.

Case 2. A 50-year-old man complained of stomach pain and anorexia. Esophageal gastric duodenal endoscopy and biopsy indicated a poorly differentiated gastric adenocarcinoma, while $\mathrm{CT}$ revealed peritoneal dissemination with multiple bone and lung metastases. Laboratory findings indicated thrombocytopenia, hypofibrinogenemia, prolonged prothrombin time and elevated FDP, leading to a diagnosis of metastatic gastric cancer with DIC. Although his thrombocytopenia and coagulopathy improved immediately after initiating SP therapy, he developed a recurrence of DIC and carcinomatous pleurisy after 3 cycles of SP therapy and died shortly thereafter.

Case 3. A 38-year-old man complained of back pain. Esophageal gastric duodenal endoscopy with biopsy showed signet ring cell carcinoma; CT revealed multiple bone metastases, lung metastasis and lymphadenopathy. Laboratory findings indicated thrombocytopenia, prolonged prothrombin time and elevated FDP. mFOLFOX6 therapy was initiated after radiation therapy for the bone metastases. However, the bolus injection of 5-FU was omitted and doses of other cytotoxic agents were reduced because of a decrease in bone marrow suppression. Recombinant thrombomodulin (rTM) was administered to improve DIC, leading to confirmed improvement 54 days after chemotherapy initiation. After 6 cycles of mFOLFOX6 therapy, he developed a recurrence of DIC and pleural effusion and, thus, received weekly paclitaxel as second-line chemotherapy. However, paclitaxel therapy failed to effectively control disease and the patient died soon after.

Case 4. A 66-year-old woman complained of anorexia, weight loss and dysphasia. Esophageal gastric duodenal endoscopy with biopsy revealed poorly differentiated adenocarcinoma and signet ring cell carcinoma. CT revealed gastric wall thickening, multiple lymphadenopathies, bone metastasis and ascites. Laboratory findings suggested DIC (thrombocytopenia, hypofibrinogenemia, prolonged prothrombin time, elevated FDP). Although thrombocytopenia improved immediately upon mFOLFOX6 therapy initiation, a CT scan revealed a new osteolytic lesion and recurrence of DIC after 8 cycles of mFOLFOX6. Weekly paclitaxel plus ramucirumab was initiated as second-line chemotherapy and found temporarily effective for coagulopathy. However, after 3 months of secondline therapy, she developed multiple liver metastases, liver function test abnormalities and recurrent DIC; the patient died shortly thereafter.

Case 5. A 35-year-old woman presented with anemia and melena. Esophageal gastric duodenal endoscopy and biopsy identified a poorly differentiated gastric adenocarcinoma and signet ring cell carcinoma, while $\mathrm{CT}$ revealed multiple bone metastases and ascites. Her platelet count at diagnosis was $80,000 / \mu 1$. Two weeks after diagnosis, she exhibited worsening thrombocytopenia with hypofibrinogenemia and a prolonged prothrombin time. mFOLFOX6 therapy was administered, although the 5-FU bolus injection was omitted because of bone marrow suppression. Thrombocytopenia and DIC improved at days 14 and 21 after therapy initiation, respectively, and a total of 12 cycles of mFOLFOX6 and two cycles of simplified leucovorin plus 5-FU were administered. 
Table I. Clinicopathological features of advanced gastric cancer patients with DIC at first-line chemotherapy.

\begin{tabular}{|c|c|c|c|c|c|}
\hline & Case 1 & Case 2 & Case 3 & Case 4 & Case 5 \\
\hline Age (years)/sex & $66 /$ male & $50 /$ male & $38 /$ male & 66/female & $35 /$ female \\
\hline Performance status (ECOG) & 1 & 1 & 4 & 1 & 2 \\
\hline Prior gastrectomy & Yes & No & No & No & No \\
\hline Histology & Por & Por & Sig & Por/Sig & Por/Sig \\
\hline HER2 status & NA & Negative & Negative & NA & Negative \\
\hline Platelet count $(/ \mu \mathrm{l})$ & 11,300 & 7,300 & 5,000 & 34,000 & 27,000 \\
\hline Serum fibrinogen $(\mathrm{mg} / \mathrm{dl})$ & 294 & 172 & 503 & 64 & 108 \\
\hline Serum FDP $(\mu \mathrm{g} / \mathrm{ml})$ & 73 & 113 & 68 & 78 & NA \\
\hline DIC score & 5 & 8 & 9 & 10 & 6 \\
\hline Bone metastasis & Yes & Yes & Yes & Yes & Yes \\
\hline Metastatic site except bone & No & Peritoneum, lung & Lung, lymph node & Liver, peritoneum, lymph node & Lymph node \\
\hline Treatment except chemotherapy & $\begin{array}{l}\text { Radiation to Th12, } \\
\text { zoledronic acid }\end{array}$ & $\begin{array}{l}\text { Zoledronic } \\
\text { acid }\end{array}$ & $\begin{array}{l}\text { Radiation to bone } \\
\text { metastasis, rTM }\end{array}$ & $\begin{array}{l}\text { Zoledronic } \\
\text { acid }\end{array}$ & $\begin{array}{l}\text { Zoledronic } \\
\text { acid }\end{array}$ \\
\hline
\end{tabular}

DIC, Disseminated intravascular coagulation; ECOG, Eastern Cooperative Oncology Group; HER2, human epidermal growth factor receptor 2; FDP, fibrin degradation product; NA, not assessed; por, poorly differentiated; rTM, recombinant thrombomodulin; sig, signet ring cell.

Table II. Outcomes of chemotherapy and clinical course.

\begin{tabular}{|c|c|c|c|c|c|}
\hline & Case 1 & Case 2 & Case 3 & Case 4 & Case 5 \\
\hline Chemotherapy regimen & $\mathrm{S}-1+\mathrm{CDDP}$ & $\mathrm{S}-1+\mathrm{CDDP}$ & mFOLFOX6 & mFOLFOX6 & mFOLFOX6 \\
\hline Improvement of DIC & Yes & Yes & Yes & Yes & Yes \\
\hline $\begin{array}{l}\text { Time of PLT recovery }(<120,000) \text { from } \\
\text { chemotherapy initiation }(\text { days })\end{array}$ & 41 & 11 & 54 & 7 & 14 \\
\hline $\begin{array}{l}\text { Time of recovery to improvement of DIC from } \\
\text { chemotherapy initiation (days) }\end{array}$ & 92 & 11 & 54 & 86 & 21 \\
\hline $\begin{array}{l}\text { Time to recurrence of DIC from } \\
\text { initial improvement (days) }\end{array}$ & 70 & 96 & None & 15 & 225 \\
\hline Adverse events (>grade 3 ) & $\begin{array}{c}\text { Anemia grade3, } \\
\text { hyponatremia grade3, } \\
\text { diarrhea grade3 }\end{array}$ & None & $\begin{array}{c}\text { Hyponatremia } \\
\text { grade } 3\end{array}$ & $\begin{array}{l}\text { Neutropenia } \\
\text { grade4 }\end{array}$ & $\begin{array}{c}\text { Neutropenia grade } 4, \\
\text { catheter-related } \\
\text { infection grade } 3\end{array}$ \\
\hline Time to treatment failure (first-line chemotherapy) & 92 & 107 & 92 & 111 & 219 \\
\hline Second- and subsequent lines chemotherapy regimens & PTX & Not done & PTX & PTX + RAM & $\begin{array}{l}\mathrm{PTX}+\mathrm{RAM} \rightarrow \\
\quad \mathrm{IRI} \rightarrow \mathrm{MF}\end{array}$ \\
\hline OS after initial chemotherapy (days)/alive or dead & 191/dead & $114 /$ dead & $131 /$ dead & $257 /$ dead & 313/dead \\
\hline
\end{tabular}

S-1, Tegafur/gimeracil/oteracil; CDDP, cisplatin; mFOLFOX6, leucovorin, fluorouracil and oxaliplatin; DIC, disseminated intravascular coagulation; PLT, platelet; PTX, paclitaxel; RAM, ramucirumab; IRI, irinotecan; MF, methotrexate and fluorouracil; OS, overall survival.

Laboratory findings following a complaint of worsening bone pain revealed elevated alkaline phosphatase, lactate dehydrogenase, carcinoembryonic antigen and carbohydrate antigen 19-9 levels; she was, thus, treated with multiple consecutive chemotherapy regimens (weekly paclitaxel plus ramucirumab, irinotecan and methotrexate plus 5-FU therapy) that led to a transient minimal hematological response (decreased transfusion frequency, reduced serum FDP levels). However, DIC and bone metastases gradually worsened and she died shortly thereafter.

\section{Discussion}

AGC with DIC is associated with a dismal prognosis; lacking cancer-specific treatment, the majority of patients die within 1-4 weeks $(6,7)$. Additionally, a majority of patients with AGC with DIC exhibit severe cytopenia, bleeding tendencies and bone metastasis and are, therefore, not good candidates for cytotoxic chemotherapy. However, we report herein five cases of AGC with DIC treated with fluoropyrimidine plus a platinum agent that suggest not only the efficacy, but also the 
safety and feasibility of chemotherapy for this disease. Previous retrospective studies have reported survival benefits of chemotherapy for AGC with DIC vs. best supportive care (BSC) $(8,9)$ with respective OS durations of 61 and 99 days vs. 9 and 16 days. In contrast, all patients, in our study, survived for $>100$ days, which further supports the efficacy of chemotherapy for AGC with DIC.

Currently, platinum-based doublet (fluoropyrimidine plus platinum) or triplet (fluoropyrimidine, platinum and taxane or anthracycline) chemotherapies comprise the standard chemotherapy regimens for AGC (10). In contrast, an optimal chemotherapy regimen for AGC with DIC has not yet been established or investigated in prospective trials. Regimens with reported efficacy against AGC with DIC include 5-FU/leucovorin therapy $(3,4)$, etoposide/ epirubicin/cisplatin/5-FU/leucovorin therapy (11) or 5-FU/methotrexate therapy (2). Each regimen exerted potent anti-DIC effect(s), with DIC improvement rates of $74-100 \%$. Despite these high DIC improvement rates, however, the median survival times ranged from 12 30 weeks, indicating a continued poor prognosis of AGC with DIC.

Little clinical information is available about the efficacy of platinum-based doublet chemotherapy for AGC with DIC. Our case series demonstrates that fluoropyrimidine (5FU/LV or S-1) plus platinum (cisplatin or oxaliplatin) chemotherapy exerts both potent anti-DIC effects $(e . g .$, all patients improved) and substantial survival time (16-44 weeks) without early death. Improvement of thrombocytopenia was achieved relatively quickly (median=14 days, range=7-41 days) after chemotherapy initiation. A previous study by Takashima et al. reported that most patients with AGC with DIC treated by methotrexate and 5-FU achieved hematological improvement within 14 days after treatment initiation (2). These early effects of chemotherapy had several effects on treatment for AGC with DIC. Namely, the improved platelet counts prevented lethal hemorrhage associated with the bleeding tendency of DIC. Furthermore, the grade $\geq 3$ hematological and nonhematological toxicities reported herein were manageable with appropriate supportive care. These results suggest that fluoropyrimidine plus platinum chemotherapy is welltolerated and effective for AGC with DIC. Although both cisplatin and oxaliplatin are considered key drugs in AGC treatment, the latter does not require hydration and is associated with less gastrointestinal toxicity and may, therefore, be advantageous for patients who are unfit to receive toxic regimens. In addition, a recent report described the efficacy of rTM for solid cancer-associated DIC (12). However, only one patient in our series received rTM and chemotherapy concurrently; accordingly, the summative or synergistic effect of chemotherapy plus rTM is unclear and requires further investigation. Recurrence of DIC developed in 4 of 5 patients $(80 \%)$ in our cases. High relapse rate of DIC after transient improvement following chemotherapy $(65 \%-85 \%)$ was reported in previous studies $(2,3,8)$. This high incidence of relapse of DIC may not be related with the chemotherapy regimen.

Our study has some notable limitations. First, this was a retrospective analysis of data from a small number of cases, with the number of patients treated with fluoropyrimidine plus platinum being too small to allow a statistical survival analysis. Second, our case series included patients with non-definitive DIC, although these patients exhibited evidence of coagulopathy and no evidence of other diseases that would mimic a diagnosis of cancer-associated DIC (e.g., severe sepsis, immunogenic or thrombotic thrombocytopenic purpura, liver cirrhosis, any thrombosis or microangiopathy). In our study, we included patients with pre- and suspected DIC after ruling out possible causes of non-cancer-associated DIC in order to describe the efficacy of chemotherapy for the extremely rare manifestation of AGC with DIC.

In conclusion, our case series suggests that fluoropyrimidine plus platinum doublet chemotherapy exhibits therapeutic efficacy, as well as an acceptable safety profile, and should be considered an initial treatment regimen for AGC with DIC.

\section{Acknowledgements}

The Authors would like to thank Enago (www.enago.jp) for the English language review.

\section{References}

1 Levi M and Ten Cate H: Disseminated intravascular coagulation. N Engl J Med 341: 586-592, 1999.

2 Takashima A, Shirao K, Hirashima Y, Takahari D, Okita NT, Nakajima TE, Kato K, Hamaguchi T, Yamada Y and Shimada Y: Sequential chemotherapy with methotrexate and 5-fluorouracil for chemotherapy-naive advanced gastric cancer with disseminated intravascular coagulation at initial diagnosis. J Cancer Res Clin Oncol 136: 243-248, 2010.

3 Huang TC, Yeh KH, Cheng AL and Hsu CH: Weekly 24-hour infusional 5-fluorouracil as initial treatment for advanced gastric cancer with acute disseminated intravascular coagulation. Anticancer Res 28: 1293-1297, 2008.

4 Yeh KH and Cheng AL: Gastric cancer associated with acute disseminated intravascular coagulation: Successful initial treatment with weekly 24-hour infusion of high-dose 5fluorouracil and leucovorin. Br J Haematol 100: 769-772, 1998.

5 Kobayashi N, Maekawa T, Takada M, Tanaka H and Gonmori $\mathrm{H}$ : Criteria for diagnosis of DIC based on the analysis of clinical and laboratory findings in 345 DIC patients collected by the Research Committee on DIC in Japan. Bibl Haematol 49: 265275, 1983.

6 Sallah S, Wan JY, Nguyen NP, Hanrahan LR and Sigounas G: Disseminated intravascular coagulation in solid tumors: Clinical and pathologic study. Thromb Haemost 86: 828-833, 2001. 
7 Pasquini E, Gianni L, Aitini E, Nicolini M, Fattori PP, Cavazzini G, Desiderio F, Monti F, Forghieri ME and Ravaioli A: Acute disseminated intravascular coagulation syndrome in cancer patients. Oncology 52: 505-508, 1995.

8 Rhee J, Han SW, Oh DY, Im SA, Kim TY and Bang YJ: Clinicopathologic features and clinical outcomes of gastric cancer that initially presents with disseminated intravascular coagulation: A retrospective study. J Gastroenterol Hepatol 25: 1537-1542, 2010.

9 Hwang IG, Choi JH, Park SH, Oh SY, Kwon HC, Lee SI, Lim DH, Lee G-W and Kang JH: Chemotherapy in advanced gastric cancer patients associated with disseminated intravascular coagulation. Cancer Res Treat 46: 27-32, 2014.

10 Waddell T, Verheij M, Allum W, Cunningham D, Cervantes A and Arnold D: Gastric cancer: ESMO-ESSO-ESTRO Clinical Practice Guidelines for diagnosis, treatment and follow-up. Ann Oncol 24: vi57-63, 2013.
11 Chao Y, Teng HC, Hung HC, King KL, Li CP, Chi KH, Yen SH and Chang FY: Successful initial treatment with weekly etoposide, epirubicin, cisplatin, 5-fluorouracil and leucovorin chemotherapy in advanced gastric cancer patients with disseminated intravascular coagulation. Jpn J Clin Oncol 30: 122-125, 2000.

12 Tamura K, Saito H, Asakura H, Okamoto K, Tagawa J, Hayakawa $\mathrm{T}$ and Aoki N: Recombinant human soluble thrombomodulin (thrombomodulin alfa) to treat disseminated intravascular coagulation in solid tumors: Results of a one-arm prospective trial. Int J Clin Oncol 20: 821-828, 2015.

Received August 26, 2016

Revised November 13, 2016

Accepted November 21, 2016 